\title{
Angiogenesis in gastric ulcers: impaired in patients taking non-steroidal anti-inflammatory drugs
}

\author{
N Hudson, M Balsitis, S Everitt, C J Hawkey
}

\begin{abstract}
Non-steroidal anti-inflammatory drug (NSAID) therapy is associated with delayed gastroduodenal ulcer healing. In rats the degree of angiogenesis (new vessel formation) within the ulcer bed correlates strongly with the extent and speed of ulcer healing and may be inhibited by NSAIDs. This study therefore assessed the vascularity of $\mathbf{3 8}$ antral gastric ulcers immunohistochemically, using CD31 a vascular endothelial cell marker, in 17 patients taking NSAIDs and 19 control patients. In the superficial granulation tissue NSAID therapy was associated with a significant reduction in the median number of capillaries (13.5 (IQR: 9.5-18) $v$ 23.5 (14-31) $(p<0.005))$, number of vessel buds $(6(4-12 \cdot 5) v 17(12-23)(p<0.005))$, and maximum vessel diameter (29 $(20.75-30.75) \quad v \quad 33.75 \quad(24-45) \quad(\mathbf{p}<0.05))$ when compared with controls. In deep granulation tissue NSAID therapy was similarly associated with a significant reduction in the number of capillaries ( 9 $(6 \cdot 5-12) v 14(9-19 \cdot 25)(p<0 \cdot 04))$, number of vessel buds $(5(3 \cdot 5-8 \cdot 5) \quad v 13(7-16 \cdot 5)$ $(\mathbf{p}<0 \cdot 01)$, and maximum vessel diameter (23 (18-20.5) v $33(21 \cdot 5-45) \quad(\mathbf{p}<0 \cdot 02))$. There were no differences in vascularity in the adjacent glandular mucosa. Impairment of angiogenesis may be an important mechanism of NSAID related delayed ulcer healing.

(Gut 1995; 37: 191-194)
\end{abstract}

Keywords: non-steroidal anti-inflammatory drugs, neovascularisation, gastric ulcer, mucosal repair.

Non-steroidal anti-inflammatory drugs (NSAIDs) are associated with an increased prevalence of gastroduodenal ulceration ${ }^{1}$ and their continued use in the presence of ulceration results in delayed healing. ${ }^{23}$ Several mechanisms contribute to ulcer healing activity including mucosal blood flow, ${ }^{4}$ cellular proliferation and migration at the ulcer edge, and maturation of granulation tissue at the ulcer base. ${ }^{5}$ Angiogenesis, the formation of new blood vessels, is an important component of granulation tissue maturation and has been seen to play a crucial part in wound healing. ${ }^{6}$ The degree of neovascularisation in experimental ulcer models correlates strongly with the extent and speed of ulcer healing. ${ }^{7-9}$ Animal studies suggest that NSAIDs impair angiogenesis. ${ }^{10}$ The aim of this study was to investigate whether NSAID therapy influenced the vascularity of granulation tissue in gastric ulcers of patients.

\section{Methods}

Histological sections of antral gastric ulcers were studied from both endoscopic biopsy specimens and surgical resection specimens. Only biopsy specimens that were well orientated and showed clearly both an ulcer edge and adjacent antral mucosa were studied. Two hundred and nine biopsy specimens were initially examined of which 38 from 36 subjects were found suitable for further analysis. Seventeen of the patients were receiving NSAIDs for at least one month before biopsy collection and there were 19 control patients. In 20 patients an antral gastric ulcer was found at upper gastrointestinal endoscopy (nine receiving NSAIDs and 11 controls). In this group informed consent was obtained before endoscopy for endoscopic biopsy of the ulcer edge with large biopsy forceps (Olympus FB13K), which permitted histological assessment of the deep granulation tissue as well as the more superficial layers (see later). Similar mucosal histology was also obtained from the edge of antral gastric ulcers in 16 patients who had undergone surgery for acute gastrointestinal haemorrhage (seven receiving NSAIDs and nine controls). Gastric ulcer was defined as a mucosal lesion with exudate of greater than 5 $\mathrm{mm}$ in diameter with a depth of greater than 2 $\mathrm{mm}$.

A detailed history with particular reference to treatment and smoking habits within the previous six months was elicited (by $\mathrm{NH}$ ) by interview, from the hospital notes, and from the patients' general practitioner records. Patients taking corticosteroids, warfarin or second line antiarthritis drugs were excluded.

The methodology of assessing vascularity was adapted from the methods previously described by Weidner et al. ${ }^{11}$ Gastric biopsy specimens were fixed in $10 \%$ formal calcium, routinely processed into paraffin wax, and one section of each was stained with haematoxylin and eosin. Serial sections were then cut at $5 \mu \mathrm{m}$ and stained immunohistochemically ${ }^{12}$ using monoclonal antibody to CD31 (Dako UK). The negative control slides were prepared without addition of primary antibody. CD31 is a membrane glycoprotein found on endothelial cells, granulocytes, monocytes, and platelets and has been found to be more specific for blood vessel endothelium than antibodies to FVIII related antigen (FVIII-rag), which has been used by other workers. ${ }^{13}$ Helicobacter pylori status was assessed on toluidine blue stained sections and graded according to the Sydney classification. ${ }^{14}$

Vessels showing positive $C D 31$ staining were counted in three high power fields at a final magnification of $\times 400$. The area of each 
Number of capillaries and vessel buds and the maximum vessel diameter ( $\mu m$ ) in superficial and deep granulation tissue and adjacent normal glandular tissue of gastric ulcers in NSAID users and non-users

\begin{tabular}{lcll}
\hline & NSAID users & Non-users & $p$ Value \\
\hline Zone 1 - Superficial granulation tissue & & & \\
No of capillaries & $13.5(9.5-18)$ & $23.5(14-31)$ & $\mathrm{p}<0.005$ \\
No of vessel buds & $6(4-12 \cdot 5)$ & $17(12-23)$ & $\mathrm{p}<0.005$ \\
Maximum vessel diameter & $29(20 \cdot 75-30 \cdot 75)$ & $33.75(24-45)$ & $\mathrm{p}<0.05$ \\
Zone 2 - Deep granulation tissue & $9(6.5-12)$ & $14(9-19 \cdot 25)$ & $\mathrm{p}<0.045$ \\
No of capillaries & $5(3.5-8 \cdot 5)$ & $13(7-16.5)$ & $\mathrm{p}<0.01$ \\
No of vessel buds & $23(18-20 \cdot 5)$ & $33(21.5-45)$ & $\mathrm{p}<0.02$ \\
Maximum vessel diameter & $13(7-20 \cdot 5)$ & $16(9.5-21)$ & $\mathrm{NS}$ \\
Zone 3 - Adjacent glandular tissue & $4(1-6)$ & $8(3.5-11.5)$ & $\mathrm{p}<0.02$ \\
No of capillaries & $31.5(19-37 \cdot 5)$ & $28.5(20.75-39)$ & $\mathrm{NS}$ \\
No of vessel buds & & & \\
Maximum vessel diameter & & & \\
\hline
\end{tabular}

Results expressed as medians with interquartile ranges.

high power field was $0.15 \mathrm{~mm}^{2}$. In suitably orientated specimens this magnification allowed the majority of each zone to be seen in a single high powered field. Vessels were identified by the presence of positively stained endothelium although no basement membrane was apparent at the light microscopic level. The vessels were defined either as capillaries - that is, endothelial lined channels with a visible lumen - or vascular buds - that is, sprouts of cells showing positive CD31 staining with the morphology of endothelial cells but with no visible lumen. Vessels were counted with the aid of a squared eyepiece graticule. Three separate high power fields were examined:

Zone 1 - the most superficial area of granulation tissue immediately below the avascular ulcer slough or regenerative epithelium and

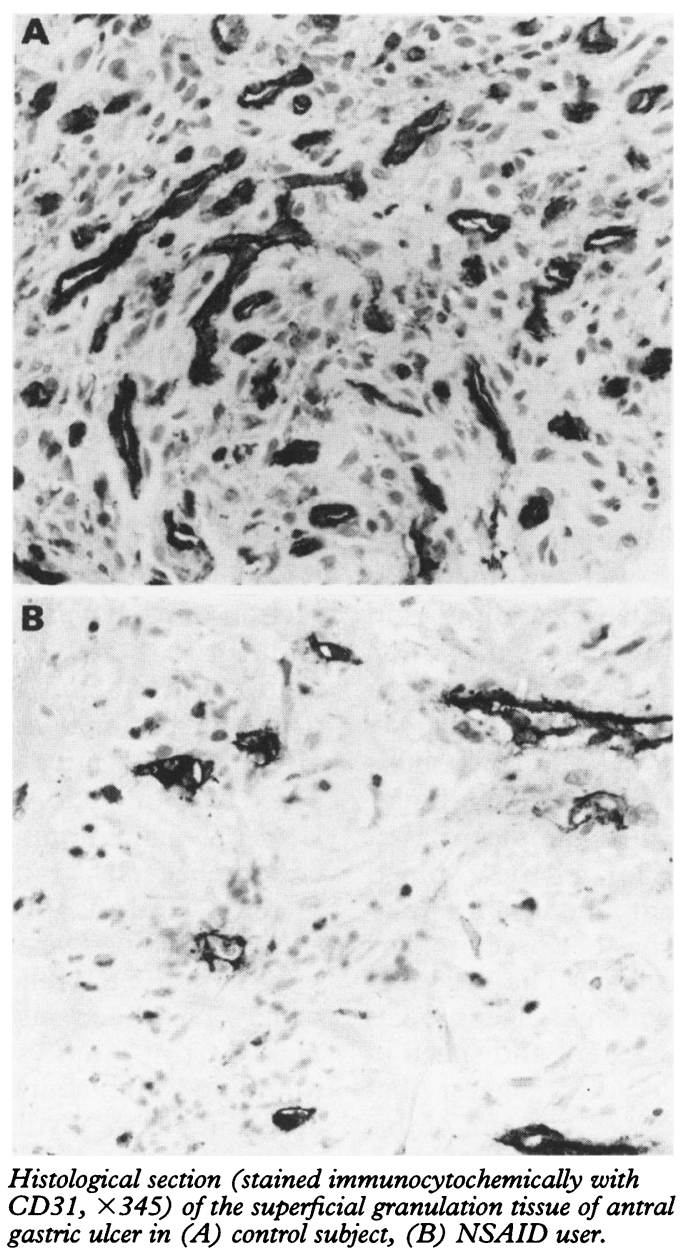

immediately adjacent to non-ulcerated glandular mucosa.

Zone 2 - granulation tissue in the high powered field immediately beneath zone 1 .

Zone 3 - glandular mucosa adjacent to the ulcer edge (that is, the high power field immediately lateral to zone 1 ).

The maximum vessel diameter in each field was also measured using a calibrated eyepiece micrometer measuring to the nearest $0 \cdot 15 \mu \mathrm{m}$. All histological assessment was carried out by a single histopathologist who was unaware of each patients' drug history. Reproducibility of histological assessment was determined by performing the counts and measurements five times in each of five cases selected at random from the group. The coefficient of variation for each feature assessed was low. The mean coefficient of variation for number of capillaries, number of buds, and maximum vessel diameter was $2 \cdot 1 \%$, $2 \cdot 8 \%$, and $4 \cdot 1 \%$ respectively.

The influence of age, sex, smoking status, $H$ pylori status, presentation (bleeding or nonbleeding), and NSAID use on each of the dependent variables was analysed by stepwise multivariate regression analysis. The Wilcoxon matched pairs signed rank test was used for pairwise comparison of the influence of NSAIDs that were identified as significant by the multivariate analysis. A $p$ value of less than 0.05 was taken as statistically significant. Histology results were expressed as medians (interquartile ranges).

\section{Results}

Patients and controls were well matched for age (mean age (SD): NSAID users $70.6(9.0)$, controls $64 \cdot 3(11 \cdot 6)$ ) sex (male:female ratio 5:12 and 8:11 respectively), and smoking status (two and three respectively). $H$ pylori colonisation was less common in NSAID users (four of 17) than in controls (nine of 19). Four subjects receiving NSAIDs had rheumatoid arthritis and the remainder osteoarthritis. None of the non-NSAID users had arthritis.

NSAID use was the only factor identified as exerting a significant influence on any of the dependent variables in the multivariate analysis. In superficial granulation tissue (zone 1) there was a significant reduction in the number of capillaries $(p<0.05)$, the number of capillary buds $(p<0.005)$, and the maximum vessel diameter $(p<0.04)$ in patients taking NSAIDs compared with controls (Table and Figure). In the deep granulation tissue (zone 2) there was a similar reduction in the number of capillaries $(p<0.045)$, the number of vessel buds $(p>0.01)$, and the maximum diameter of capillaries $(p<0.02)$ in the NSAID patients. In contrast there was no reduction in the number or maximum diameter of capillaries in the adjacent glandular mucosa (zone 3) although the number of buds were reduced in those patients taking NSAIDs $(p<0.02)$.

\section{Discussion}

The results of this study are compatible with the proposition that NSAIDs inhibit 
neovascularisation in the granulation tissue of the gastric ulcer bed and hence impair ulcer healing. Age, sex, and smoking status did not seem to influence neovascularisation making it unlikely that the association with drug use is a surrogate for other influences on ulcer healing. Similarly $H$ pylori colonisation and associated mucosal inflammation did not seem to influence neovascularisation. While ulcers that present with upper gastrointestinal haemorrhage could in theory have different characteristics from those presenting at endoscopy we found no differences in the vascularity of granulation tissue between these groups.

Gastric ulcer healing entails several distinct repair mechanisms. Epithelial cell proliferation and migration from the ulcer edge across the ulcer bed is accompanied by maturation of granulation tissue beneath the ulcer base. Within this tissue vascular endothelial cells form new capillaries to restore the microvasculature while fibroblasts restore the lamina propria. ${ }^{5}$ Angiogenesis is defined as the formation of new blood vessels. There is now evidence of its importance in healing and repair mechanisms at a variety of sites. Suppression of angiogenesis causes retardation of cutaneous wound healing. ${ }^{6}$ Folkman et al have shown that oral basic fibroblastic growth factor, a potent vascular endothelial mitogen found in stomach and duodenum stimulates neovascularisation of the ulcer bed but not in adjacent normal mucosal epithelium and is associated with acceleration of duodenal ulcer healing in rats. ${ }^{78}$ Enhanced vascularity of granulation tissue at the ulcer margin after basic fibroblastic growth factor is given and subsequent accelerated ulcer healing has also been seen in rat gastric ulcers. ${ }^{9}$

Several lines of evidence suggest that NSAID therapy is associated with inhibition of repair mechanisms in gastroduodenal ulceration. Continued NSAID therapy in the presence of ulceration is associated with delayed ulcer healing both in animals and humans. ${ }^{2} 315$ Indomethacin also inhibits epithelial cell regeneration within gastric glands at the ulcer edge in humans. ${ }^{16}$ Further experimental studies of gastric ulcers induced by acetic acid have shown that indomethacin impairs angiogenesis within the granulation tissue of ulcers. ${ }^{10}$ The mechanism of inhibition of angiogenesis by NSAIDs remains unclear although in experimental ulcers exogenous prostaglandin $\mathrm{E}_{1}$ reverses the detrimental effects of indomethacin on angiogenesis. ${ }^{17}$ Prostaglandin $\mathrm{E}_{2}$ alone has been shown to be a potent stimulator of angiogenesis in the chorioallantic membrane of chicken embryos. ${ }^{18}$ Whether NSAIDs also act by inhibition of the actions of mucosal growth factors within the stomach is unknown although recent evidence suggests that indomethacin may interfere with binding of exogenous basic fibroblastic growth factor to the ulcer base. ${ }^{9}$

The conclusions drawn from this study depend on both the specificity of the endothelial marker CD31 and the accuracy of the morphometric measurements. Anti-CD31 monoclonal antibody reacts with a membrane glycoprotein found in endothelial cells and platelets. In cryostat and blood smears the antibody labels endothelium as the only organised or tubular structure, or both, labelled by the antibody although platelets and occasional plasma cells can be seen to stain. ${ }^{13}$ In formalin fixed paraffin wax embedded tissue, however, platelets and leucocytes do not stain with this antibody and this seems restricted to endothelial cells. ${ }^{13}$ Staining is more consistent and specific than found with either FVIII-rag or the lectin Ulex europaeus agglutinin lectin and in contrast with FVIII-rag CD 31 does not stain lymphatic endothelium. ${ }^{13}$ We showed low observer variability in assessing the number, type, and diameter of blood vessels. Not all samples could be subjected to a variability study because comparatively small areas of mucosal tissue were suitable for analysis when acquired from ex vivo endoscopic biopsy specimens. Such restrictions, however, may be expected to exaggerate variability between samples and make significant differences between groups less rather than more probable.

In conclusion this study suggests that in humans NSAIDs are associated with a reduction in vascularity of the ulcer bed and by inference inhibition of angiogenesis in the granulation tissue of gastric ulcers, which seems to be an important factor contributing to ulcer healing. Whether this effect is mediated in whole or part by impairing the synthesis or activity of growth factors and whether this is prostaglandin dependent requires further study.

We are grateful to $\mathrm{Mr} P$ Riley of Cripps Computing Centre University of Nottingham for statistical advice.

Part of this work was presented at the British Society of Gastroenterology, Autumn Meeting, London 1991 and published in Gut 1991; 32: A1256.

1 Hawkey CJ. Non-steroidal anti-inflammatory drugs and peptic ulcers. Facts and figures multiply, but do they add up? BMF 1990; 300: 278-84.

2 Lancaster-Smith MJ, Jaderberg MR, Jackson DA Ranitidine in the treatment of non-steroidal anti-inflammatory drug associated gastric and duodenal ulcers. Gut 1991; 32: 252-6.

3 Manniche C, Malchow-Molloer A, Anderson JR, Pedersen C, Hanson TN, Jess P. Randomised study of the influence of non steroidal anti-inflammatory drugs on the treatment of peptic ulcer in patients with rheumatoid arthritis. Gut 1987; 28: 226-9.

4 Hirose H, Takeuchi K, Okabe S. Effect of indomethacin on gastric mucosal blood flow around acetic acid-induced gastric ulcers in rats. Gastroenterology 1991; 100: 1259-65.

5 Tarnawski A, Douglass TG, Stachura J, Krause WJ. Quality of gastric ulcer healing: histological ultrastruc-
tural assessment. Aliment Pharmacol Therap 1991; 5 tural assessment.

6 McGrath MH, Emery JMI. The effect of inhibition of angiogenesis in granulation tissue on wound healing and the fibroblast. Ann Plast Surg 1985; 15: 106-19.

7 Folkman J, Szabo S, Stovroff M, McNeil P, Li W, Shing Y. Duodenal ulcer: discovery of a new mechanism and development of angiogenic therapy that accelerates healing. Ann Surg 1991; 214: 414-27.

8 Szabo S, Folkman J, Vattay P, Morales RE, Pinkus GS, Kato K. Accelerated healing of duodenal ulcers by oral administration of a mutein of basic fibroblastic growth factor in rats. Gastroenterology 1994; 106: 1106-11.

9 Konturek SJ, Brzozowski T, Majka J, Szlachcic A, Bielanski $\mathrm{W}$, Stachura J, et al. Fibroblast growth factor in gastroprotection and ulcer healing: interaction with sucralfate. Gut 1993; 34: 881-7.

10 Tarnawski A, Hollander D, Stachura J, Sarfeh IJ, Gergely $\mathrm{H}$, Krause WJ. Angiogenic response of gastric mucosa to ethanol injury is abolished by indomethacin. Gastroenterology 1989; 96: A505.

11 Weidner N, Semple JP, Welch WR, Folkman J. Tumour angiogenesis and metastasis - correlation in invasive breast carcinoma. N Engl $₹ \mathrm{Med}$ 1991; 324: 1-8.

12 Mayer RJ, Walker JH. Immunochemical methods in cell and molecular biology. London: Academic Press, 1987. 
13 Parums DV, Cordell JC, Micklem K, Heryit AR, Gatter KC, Mason DY. JC70: a new monoclonal antibody that detects vascular endothelium associated antigen on routinely processed tissue sections. 7 Clin Pathol 1990; 43: routinely processed tissue sections. F Clin Pathol 1990; 43:

14 Price AB. The Sydney system: histological division. $f$ Gastroenterol Hepatol 1991; 6: 209-22.

15 Wang JY, Yamasaki S, Takeuchi K, Okabe S. Delayed healing of acetic acid induced gastric ulcers in rats by indomethacin. Gastroenterology 1989; 96: 393-402.
16 Levi S, Goodlad RA, Lee CY, Stamp G, Walport MJ, Wright NA, et al. Inhibitory effect of non-steroidal antiinflammatory drugs on mucosal cell proliferation associinflammatory drugs on mucosal cell proliferation associ-

17 Tarnawski A, Hollander D, Stachura J, Sheffield M, Tarnawski A, Hollander D, Stachura J, Sheffield M,
Gergely H, Krause WJ. Angiogenic response of damaged gastric mucosa - a prostaglandin mediated mechanism. Gastroenterology 1990; 98: A136.

18 Form DM, Auerbach R. PGE 2 and angiogenesis. Proc Soc Expt Biol Med 1983; 172: 214-8. 